\title{
Combining Hough Transform and Optimal Filtering for Efficient Cosmic Ray Detection with a Hadronic Calorimeter
}

\author{
Luciano M. de A. Filho* \\ Federal University of Rio de Janeiro \\ E-mail: Lucianomelps.ufrj.br \\ José M. de Seixas \\ Federal University of Rio de Janeiro \\ E-mail: seixasdps.ufrj.br
}

\begin{abstract}
The hadronic calorimeter of ATLAS, TileCal, provides a large amount of readout channels (about 10,000). Therefore, track detection may be performed by TileCal when cosmic muons cross the detector. The muon track detection has extensively been used in the TileCal commissioning phase, for both energy and timing calibrations, and it will also be important for background noise removal during nominal LHC operation. This work presents a cosmic ray detection algorithm based on TileCal information. The algorithm employs the Hough Transform to map the data from activated calorimeter cells into a parameter (straight-line track) space, in which detection is effectively performed. Due to intrinsic low signal-to-noise ratio for cosmic ray detection with TileCal, a preprocessing algorithm based on a matched filter operating over TileCal time sampled signals is implemented to improve detection efficiency. Experimental data show that the proposed method outperforms the straight-line fitting methods previously used in TileCal.
\end{abstract}

XII Advanced Computing and Analysis Techniques in Physics Research

November 3-7 2008

Erice, Italy

\footnotetext{
*Speaker.

$\dagger$ This work was supported by CNPq, FAPERJ, FINEP and CAPES (Brazil) and the European Union through the HELEN project. The authors would like to thank our colleagues from the ATLAS TileCal collaboration for providing the data and for fruitful discussions concerning this work.
} 


\section{Introduction}

The ATLAS detector [ $[$ ] is in the final phase of a detailed test and calibration process. For that, tests using Cosmic Rays (CR) as signal source are being performed in different sub-detectors. An important task is the measurement of the energy left by a CR when it crosses the calorimeter. In order to achieve this goal, an efficient track detection algorithm, looking at the activated calorimeter cells, should be implemented. In this way, one can verify whether the track has coherently been reconstructed, look for potential problems and test calorimeter uniformity.

The method previously used as the base line cosmic ray detection algorithm in the hadronic calorimeter (TileCal) aimed at minimizing the sum of quadratic distances among activated cells and the desired tracks [】], using Least Square Methods (LSM). However, the LSM has an inherent limitation: the sensitivity to outliers [B]]. In other words, although LSM manipulates efficiently measurement errors which appear as small fluctuations in the data set, it does not tolerate outliers. This kind of noise, even in small quantity, produces residues, generating wrong track adjustments. Besides, the LSM is not able to detect multiple tracks by itself.

This work presents an alternative algorithm to detect cosmic rays in the TileCal, using the Hough Transform (HT) method [ [ 4 ]. This technique has extensively been used to detect straightline segments in highly noisy images and was here adapted to detect tri-dimensional tracks. HT proved to be able to deal with the noise contamination that makes cosmic ray track detection in TileCal quite difficult. Besides, HT can detect multiple track in a more direct way.

Due to the low signal-to-noise ratio of the TileCal for cosmic rays, we have also used a matched-filter [1] to identify the CR activated cells in the TileCal. The matched-filter (MF) operates over the time sampled signals of individual calorimeter cells and produces higher signal detection efficiency with respect to standard energy cut procedures. This reduces the number of outliers, increasing the muon track detection efficiency.

\section{The ATLAS detector}

As one of the main LHC [目] detectors, ATLAS will explore the fundamental nature of matter and the basic forces that shape our universe. The detector has a total length of $42 \mathrm{~m}$ and a radius of $11 \mathrm{~m}$ (see Figure प).

The Inner Detector $[\mathrm{l}]$ extends to a radius of $1.2 \mathrm{~m}$, and is $7 \mathrm{~m}$ in length along the beam pipe. Its basic function is to track charged particles and, thus, measuring their momentum. The calorimeter [8] is situated outside the solenoidal magnet that surrounds the inner detector and comprises two sections: an electromagnetic [0] and a hadronic [U]] part. Their purpose is to measure the energy of incoming particles by total absorption [W]. The muon spectrometer is an extremely large detection system, extending from a radius of $4.25 \mathrm{~m}$ around the calorimeters out to the full radius of the detector $(11 \mathrm{~m})[\mathbb{[} 2]$. Its tremendous size is required to accurately measure the momentum of muons.

Due to the extremely high interaction rate and the huge background noise generated by LHC, ATLAS requires the design of a sophisticated on-line triggering system, for which calorimeters play an important role. In ATLAS, the triggering system operation is split into three cascaded 


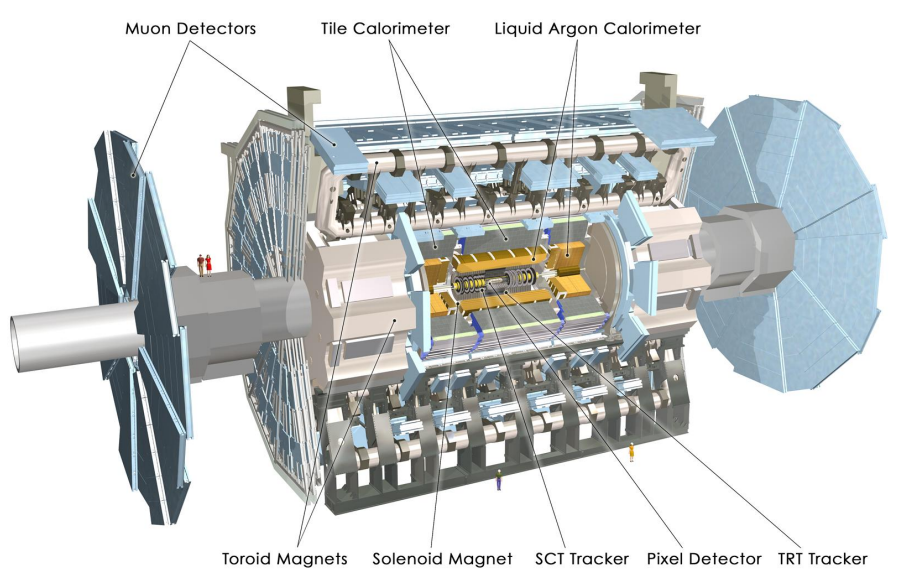

Figure 1: The ATLAS detector and its main subsystems.

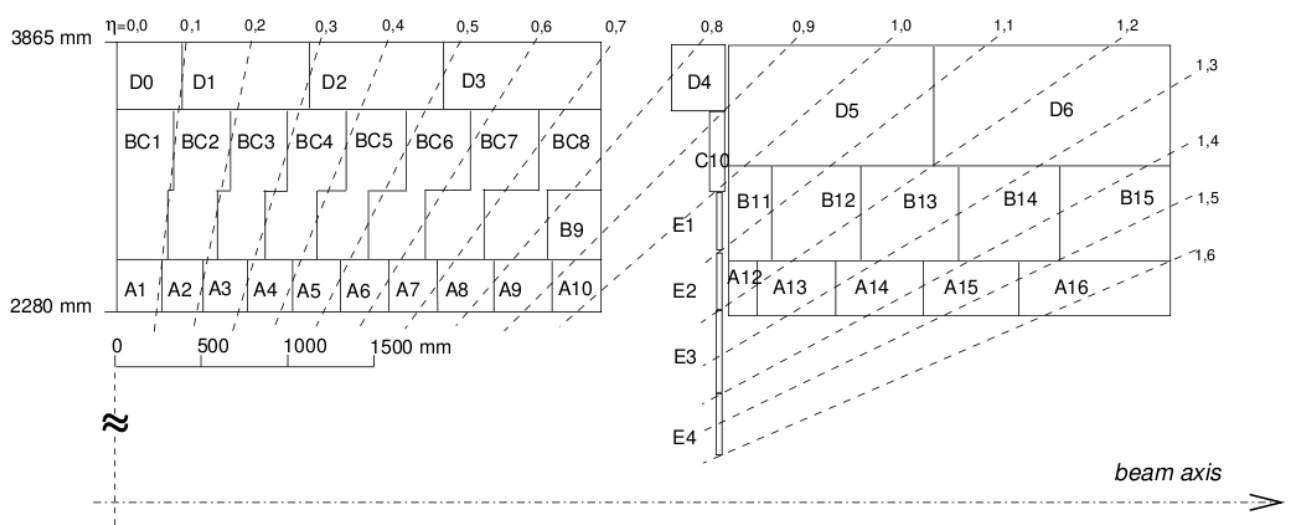

Figure 2: TileCal segmentation.

levels and should reduce dramatically the background noise rate, providing an output frequency of the order of $100 \mathrm{~Hz}$ [एँ3]].

\subsection{TileCal segmentation}

The TileCal absorbs energy from particles that pass through the EM calorimeter, which are primarily hadrons. The energy-absorbing material is steel and scintillating tiles sample the deposited energy.

The TileCal comprises three cylindrical parts: one barrel, which covers the rapidity range $|\eta|<1.0$, and two extended barrel wheels, covering the rapidity range $0.8<|\eta|<1.7$ on either side of the barrel. The barrel and the two extended barrels are separated by a gap of $600 \mathrm{~mm}$ to provide space for liquid argon distribution pipes. Each of the sub-detectors is composed of 64 azimuthal wedge-shaped modules. Figure $\square$ shows the TileCal segmentation. Each Calorimeter cell is coupled to two PMTs for readout redundancy. 


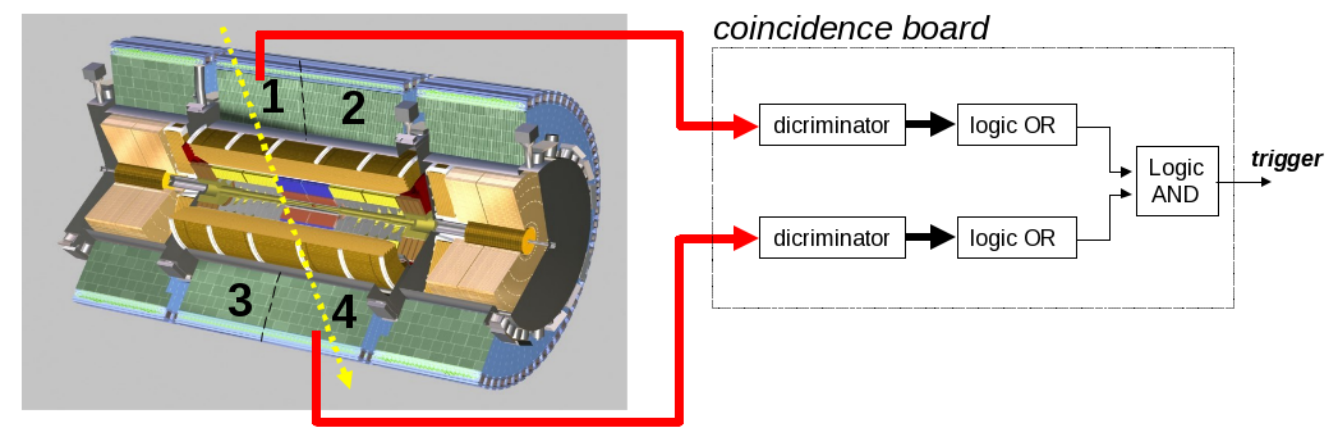

Figure 3: Triggering cosmic rays with TileCal.

\section{Commissioning with cosmic rays}

For the calorimeter subsystem, the goal of cosmic ray commissioning [[14]] is twofold: calibration and performance evaluation, ensuring its readiness for LHC turn-on. In the beginning of the commissioning phase, the stand-alone cosmic data acquisition has been accomplished by a coincidence board [ㄷ]]. Figure [3] shows the setup. The straight-line represents a cosmic muon crossing the center of the detector. For triggering, coincidence from both top and bottom parts of the Tile$\mathrm{Cal}$ is required. When a trigger signal is generated, data from both hadronic and electromagnetic calorimeters can be recorded with full granularity. The coincidence board uses compacted information from TileCal, obtained from its trigger towers [ए]]. These towers correspond to the analog sum of all PMTs within $\Delta \eta<0.1$, for each module. In Figure $\square$, one can identify, for instance, that the first tower corresponds to the sum of signals from A1, BC1 and D0 cells.

Cosmic ray muons can be used to check various detector parameters. While the cell by cell statistics might not be sufficient, it will be possible to sum similar cells (along the $\phi$ direction) and perform physics signal shape studies, as well as amplitude and time inter-calibrations. The problematic channels, such as having HV different from nominal voltage, dead readout channels etc, can be identified, and proper treatment of these channels can be investigated.

\section{Cosmic ray detection algorithms}

In order to detect cosmic rays in TileCal, two different techniques were combined. The track detection itself is performed by Hough Transform (HT) methods. To identify which calorimeter cells would be used by the HT algorithms (candidate cells having significant energy deposition), signal detection using matched-filters is performed, looking at the time-digital samples of individual cells.

\subsection{Track detection with Hough Transform}

Hough Transform (HT) is a well known technique for image processing aiming at detecting straight-lines in noisy and missing information environments [ㅍ] . HT maps the input data space into a parameter space of either linear or sinusoidal functions. When the mapping functions is

$$
\rho=x \cos \theta+y \sin \theta,
$$



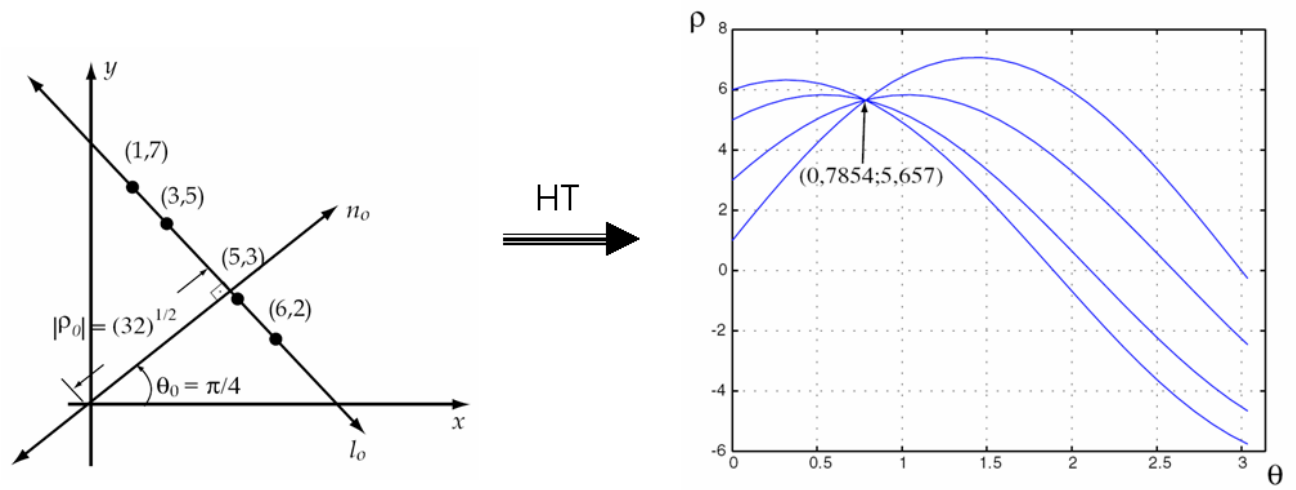

Figure 4: Hough Transform applied to points which belong to the same straight-line.

data points in the $x-y$ plane located on the straight-line $\rho_{0}=x \cos \theta_{0}+y \sin \theta_{0}$ are mapped onto curves in the $\rho-\theta$ space (see Figure $\Psi$ ), with all curves passing trough $\left(\rho_{0}, \theta_{0}\right)$.

In order to detect the original straight-lines in the data space, both $\rho$ and $\theta$ axes have to be quantized and hence a two-dimensional accumulator array is constructed in the $\rho-\theta$ plane. A search is then performed to locate peaks in the accumulator.

The HT approach as presented above has an extensive computational cost. Some variations of the standard HT method have been proposed in order to reduce the computational effort. Among them, the Local Hough Transform (LHT) [ए8] and the Adaptive Hough Transform (AHT) [एप] are used in this work.

In the standard HT, a single point in the data space generates several points in the parameter space. In contrast, the Local HT (LHT) looks for the best straight-line which can be generated by a given point set in the data space. In this way, LHT maps a pair of points of the data space in a single point in the parameter space. If two different pairs of points belong to the same line in the data space, they will produce a increment of the same accumulator cell. Then, as in the standard HT case, a peak in the accumulator represents a high probability to have a straight-line in the data space.

For the Adaptive Hough Transform (AHT), the main idea consists in applying the transformation several times for a given data space, doing a zoom in the accumulator, at the peak area, for each iteration. Thus, even for an accumulator with low amount of cells, a high resolution detection can be performed after a given number of iterations.

\subsubsection{Implementation in TileCal}

In order to implement a cosmic ray track detection in TileCal using HT, some steps should be executed before performming the track detection itself. The HT algorithm is applied to each cosmic ray triggered event. The whole procedure is listed bellow:

1. Select only activated cells, either applying energy cuts or using matched filters for signal detection (see next subsection).

2. Replace cell geometry by their $(x, y, z)$ center point positions. 

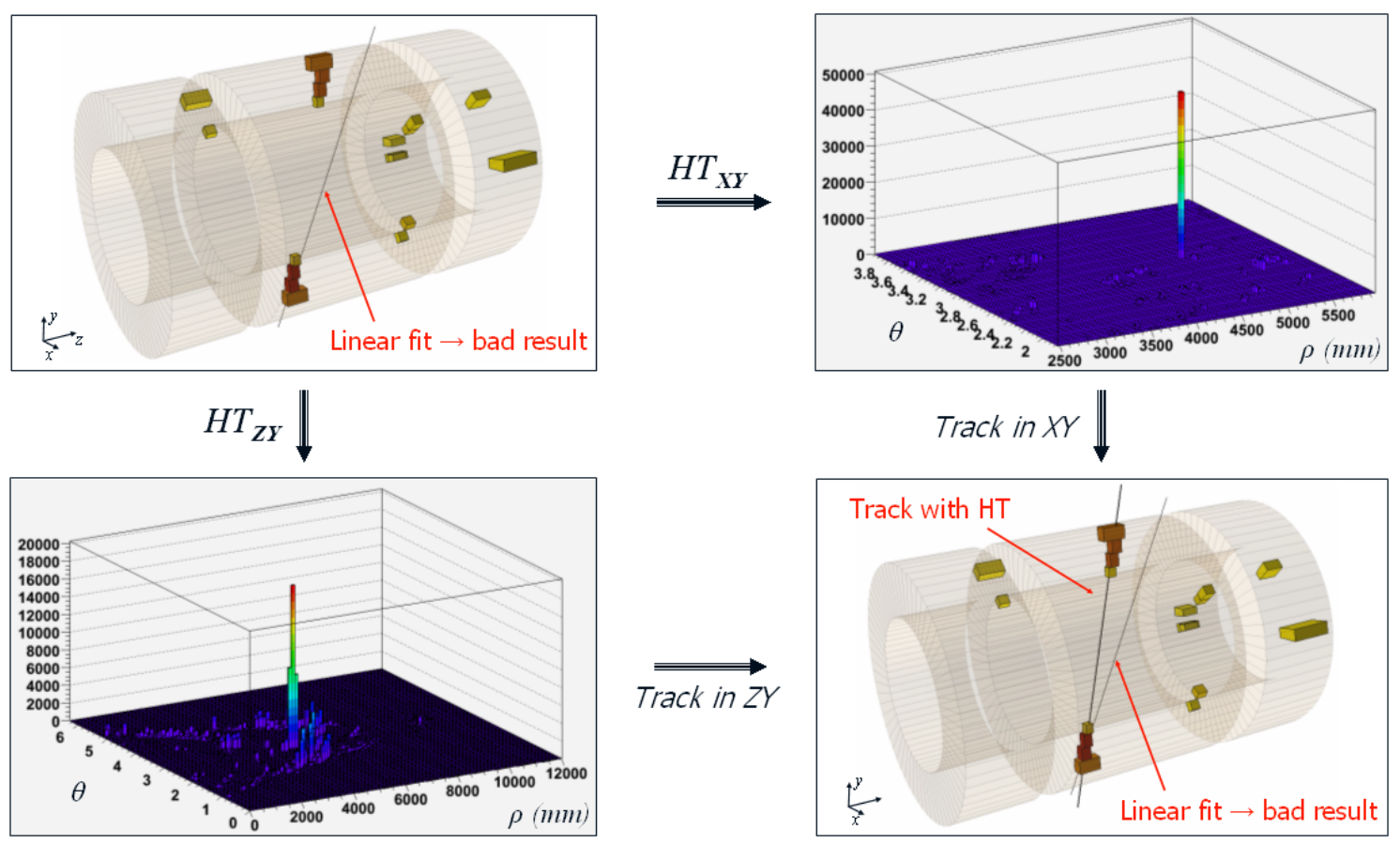

Figure 5: Example of a cosmic ray detection using HT. Track detection from a straight-line fitting is also shown for comparison.

3. Project center points of activated cells onto the XY and ZY planes.

4. Find straight-lines in each plane using the HT method.

5. Merge the straigth-line segments from each plane in 3D tracks.

The implemented HT algorithm was a combination of LHT with two AHT iterations. Cell energy information is used as a weighting factor, in order to get fine track adjustment. Figure [5 shows an example of cosmic ray detection in the TileCal using the HT. Activated cells (energy deposition above $100 \mathrm{MeV}$ ) are shown in a 3-D view of the detector. A fitting result for the activated cells is also shown for comparison. One can notice that the fitting results in a wrong track due to the presence of noise cells (outliers), which are relatively far from the actual cosmic muon track.

\subsection{Signal detection with matched filters}

In order to perform track detection, one should first identify which calorimeter cells have been activated by the cosmic ray interaction. The standard approach comprises a cut above noise level to be applied to the energy deposition estimation for each calorimeter cell. Typically, in TileCal, above $100 \mathrm{MeV}$ a given cell is considered to be activated.

Here, we consider a matched filter based algorithm. The matched filter will be designed using the digitized time signal sampled from each calorimeter cell (one digitized signal per PMT), using the TileCal analog-to-digital converter. For each triggered event, 7 samples of each PMT time signal (the pulse shape) are recorded, using a $40 \mathrm{MHz}$ sampling rate. 
The MF approach is based on a hypothesis test. In other words, the detection system has to decide between two possible hypothesis: only noise $\left(H_{0}\right)$ or signal and additive noise are present in the receiving end of the detection system $\left(H_{1}\right)$. The decision rule can be summarized by the likelihood equation

$$
\frac{f_{\mathbf{L} \mid H_{1}}\left(\mathbf{l} \mid H_{1}\right)}{f_{\mathbf{L} \mid H_{0}}\left(\mathbf{l} \mid H_{0}\right)} \underset{H_{0}}{\stackrel{H_{1}}{\gtrless}} \gamma
$$

where $f_{\mathbf{L} \mid H_{1}}\left(\mathbf{l} \mid H_{1}\right)$ and $f_{\mathbf{L} \mid H_{0}}\left(\mathbf{I} \mid H_{0}\right)$ are the conditional Probability Density Functions (PDF) for a given outcome $\mathbf{L}$ of $N$ samples, depending whether the valid hypothesis is either $H_{1}$ or $H_{0}$, respectively. Thus, for each calorimeter cell, $H_{1}$ (cell selected) is chosen if the likelihood ratio is above $\gamma$, otherwise $H_{0}$ is considered correct (noisy cell). The constant $\gamma$ can vary, depending on costs assigned to a decision between one of the two hypothesis [ [వ]. Time-digitized noise signals were obtained from pedestal runs.

\subsubsection{PDF estimation}

Based on cosmic ray simulation, Figure 6 shows the probability distribution of each time sample, in terms of Analog-to-Digital (ADC) counts, for both hypotheses $H_{1}$ and $H_{0}$ (Signal and Background, respectively). In order to consider only low signal-to-noise ratio hits, cells with energy deposition higher than $500 \mathrm{MeV}$ were discarded for PDF estimation.

As a first PDF estimation approach, the samples will be considered statistically independent. In this way, the PDF will be the product of individual probability distributions. Even though statistical independence may not be a realistic assumption here, this simple MF approach produces satisfactory detection efficiency results, as will be shown in Section 5. Further improvements can be achieved by performing some preprocessing techniques, where the samples become either statistically independents or, at least, uncorrelated (see Section 4.2.3).

\subsubsection{Noise whitening}

For MF design, the additive noise should be white [[20]. The left side of Figure $\square$ shows the covariance matrix for the TileCal digital noise samples, where one can see some correlation among neighbor samples. On the right, it is shown in Figure $\square$ the covariance matrix after noise whitening transformation [ [R]]. The used whitening method consists to a decorrelation process, using PCA (see next section), followed by normalization of each component, in order to get unitary variancy [22].

\subsubsection{Preprocessing}

The $H_{1}$ individual distribution can become uncorrelated, performing Principal Component Analysis - PCA [23] . PCA projects the distributions onto an orthogonal base, composed by the eigenvectors of the signal covariance matrix. The variances of the new distributions will be the correspondent eigenvalues. Figure $\mathrm{Q}$, left, shows the covariance matrix of $H_{1}$ after whitening transformation. On the right, the eigenvalues of this matrix are plotted, sorted out by amplitude. One can notice that the signal energy is concentrated on the three first components only. Thus, this transformation allows to perform a dimensional reduction from 7 to 3 components, without loosing significant information. Besides, since PCA is just a orthonormal transformation (rotation) the noise $\left(H_{0}\right)$ will still be white in this new base. 

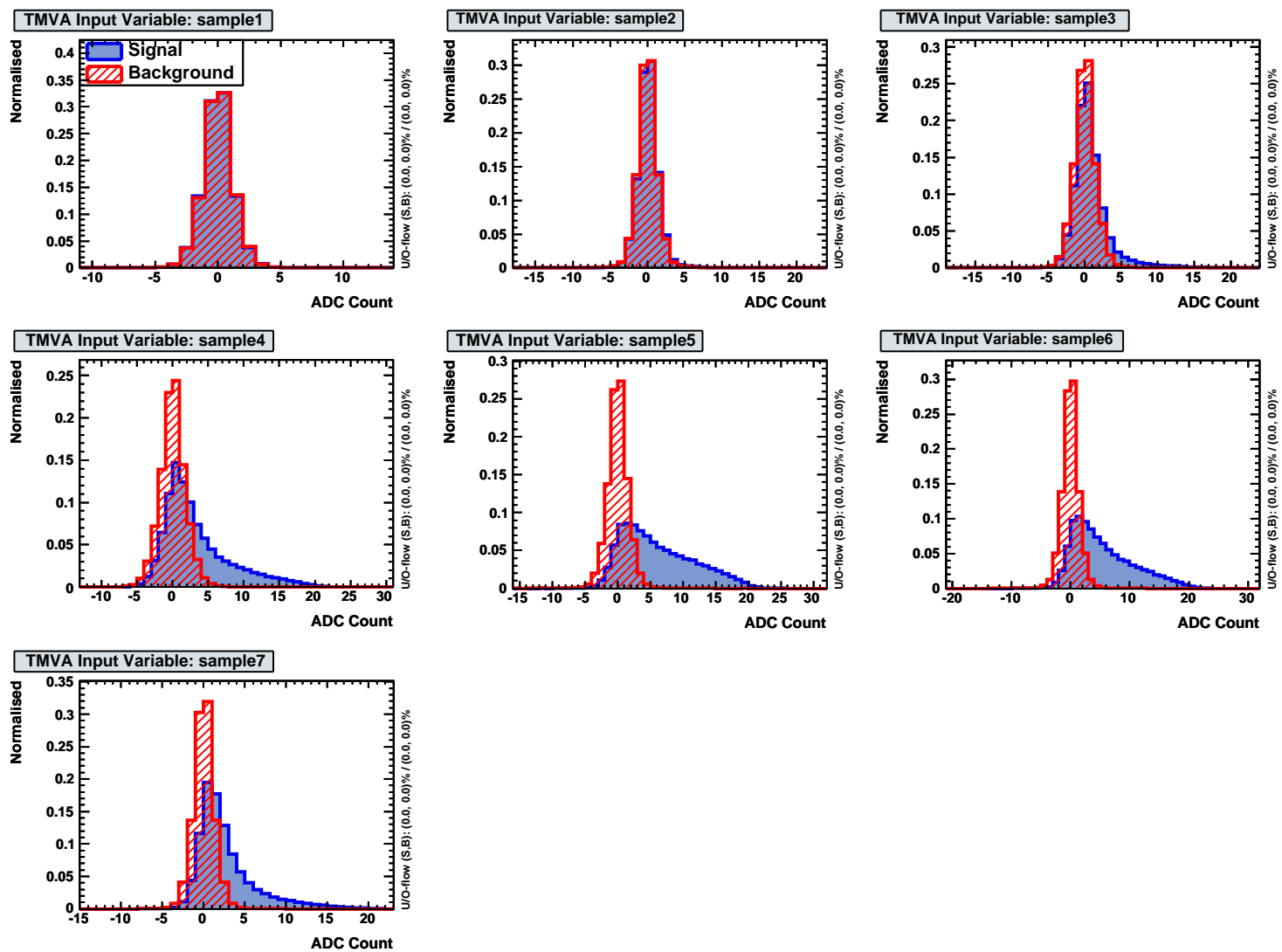

Figure 6: Probability distributions, in ADC counts, of the seven TileCal time samples, for both $H_{1}$ and $H_{0}$ hypotheses.
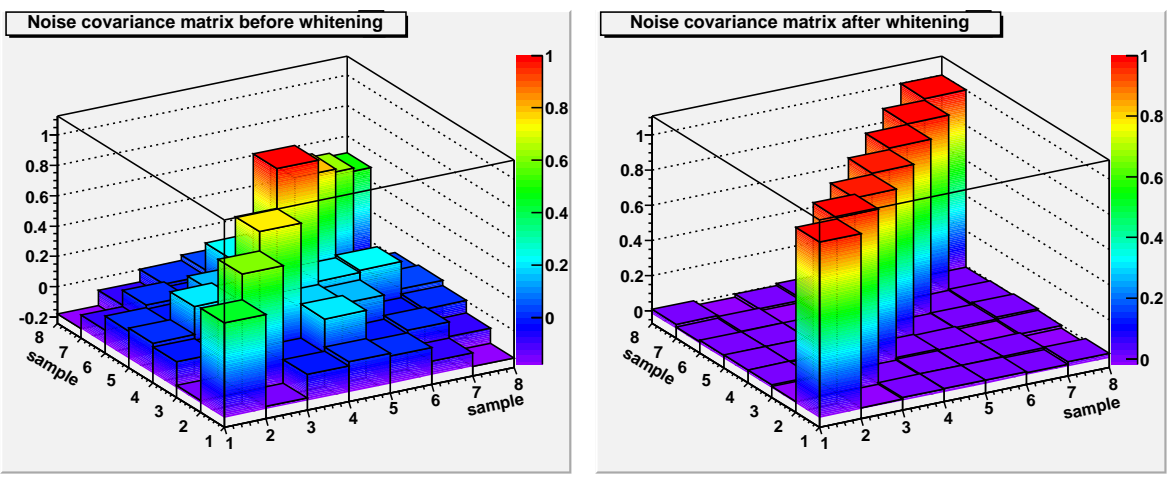

Figure 7: Noise covariance matrix before and after the whitening transformation. 

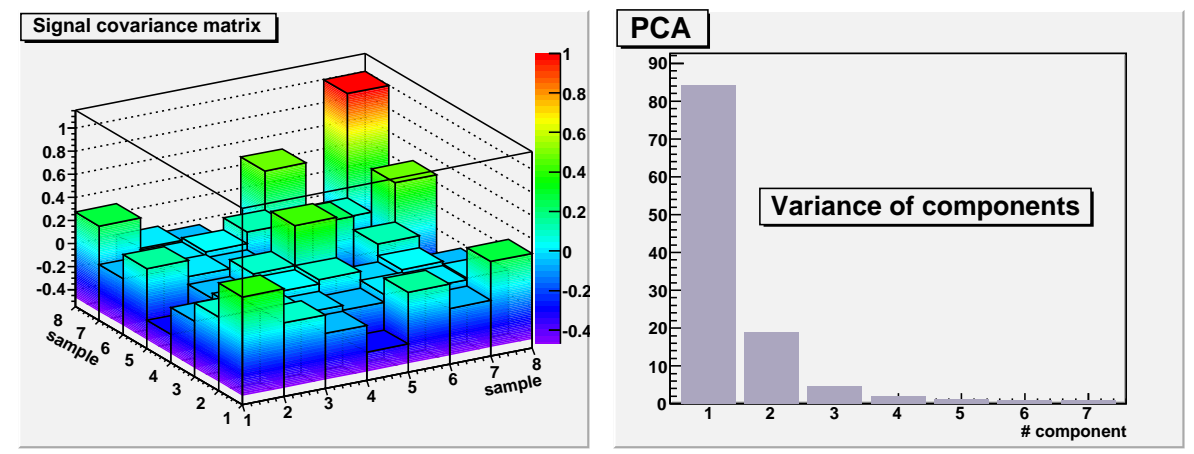

Figure 8: Covariance matrix of $H_{1}$. After PCA, signal energy is concentrated in the three first components.
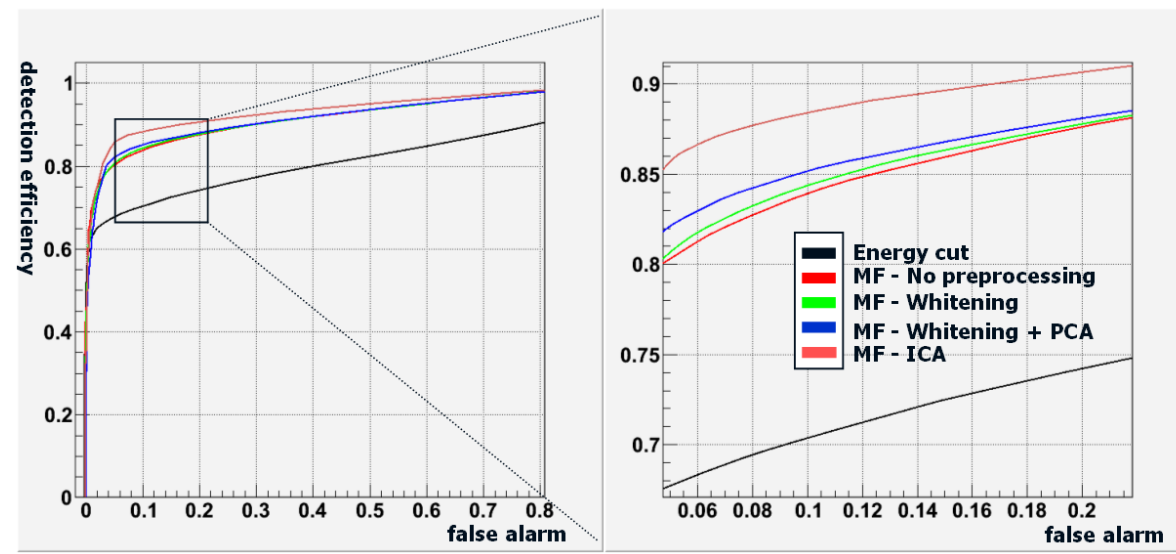

Figure 9: Detection efficiency versus false alarm. All MF approaches give better results than the energy cut method.

Since the sample distributions are not Gaussian (see Figure 6), PCA and whitening transformations (decorrelation) are not enough to get statistical independence. In order to achieve this, Independent Component Analysis (ICA) [22] may be applied. ICA is based on the central limit theorem: combinations of nongaussian variables are more Gaussian than the original ones. Thus, ICA transformation tries to maximize the nongaussianity of the components. The nongaussianity measurements was based on negentropy and the method to achieve the independents components was the FastICA algorithm [22].

\section{Results}

Figure $\mathbf{Q}$ shows the ROC (Receiver Operating Characteristics) curve [[]] for different signal detection approaches. The methods based on matched filters outperform the simple energy cut method. It shouls be noticed that PCA compaction preserves the detection performance, although only 3 components in the orthogonal PCA base are considered. Among the MF methods, the one with ICA preprocessing has the best performance. 


\section{Conclusion}

We have presented a combination of Hough Transform and Optimal Filtering techniques in order to detect cosmic rays in the hadronic calorimeter of ATLAS. The Hough Transform was adapted to detect cosmic ray tracks, using information of activated cells. The Matched Filter was used to select the activated cells, looking at their time-digitized signals. We have shown that this approach has a higher detection efficiency with respect to energy cut methods. Analysis of energy deposition of cosmic rays in TileCal has shown the good performance of the algorithms.

\section{References}

[1] The ATLAS Collaboration, The ATLAS Experiment at the CERN Large Hadron Collider, Journal of Instrumentation, JINST 3 S08003, 2008.

[2] https://twiki.cern.ch/twiki/bin/view/Atlas/TileMuonFitter (accessed on December 2008).

[3] G. H. Golub, C. F. Van Loan, Matrix Computation, Johns Hopkins Univ. Press, 1984.

[4] P. Hough, Method and mean for recognizing complex patterns, United States Patent 3069654, 1962.

[5] H.L.Van Trees. Detection, Estimation, and Modulation Theory, Part I, Wiley, 2001.

[6] L. Evans and P. Bryant. LHC Machine, Journal of Instrumentation, JINST 3 S08001, 2008.

[7] Eduardo Ros. ATLAS inner detector, Nuclear Physics B - Proceedings Supplements, v. 120, pp. 235-238, 2003.

[8] The ATLAS Collaboration. Calorimeter Performance, Technical Design Report, CERN/LHCC/96-40, 1997.

[9] P. Perrodo. The ATLAS liquid argon calorimetry system, Proceedings of ICHEP, pp. 909-912, 2002.

[10] The ATLAS Collaboration. Tile Calorimeter, Technical Design Report, CERN/LHCC/96-42, 1996.

[11] R. Wigmans, Calorimetry - Energy Measurement in Particle Physics, Oxford University Press, 2000.

[12] S. Palestini. The Muon Spectrometer of the ATLAS Experiment, Nuclear Physics B, v. 125, pp. 337-345, 2003.

[13] S. Falciano. The ATLAS Level-l and Level-2 trigger, Nuclear Instruments and Methods in Physics Research A, v. 384, pp. 136-142, 1996.

[14] Gerolf Schlager. The status and performance of the ATLAS hadronic tile calorimeter, Nuclear Instruments and Methods in Physics Research A, v. 581, pp. 393-396, 2007.

[15] K.Anderson, J.Pilcher, H.Sanders, F.Tang and R.Teuscher. Stand-alone Cosmic Ray Trigger Electronics for the ATLAS Tile Calorimeter. 10th Workshop on Electronics for LHC and future Experiments, Boston, USA, pp. 327-331, 2004.

[16] A. Cerqueira, J. M. Seixas, L. P. Caloba. Analog system for building the first-level triggering signal provided by the ATLAS hadronic calorimeter of ATLAS detector. Nuclear Instruments and Methods in Physics Research A, v. 570, pp. 117-125, 2007.

[17] J. Illingworth. A survey of the Hough Transform, Computer Vision, Graphics and Image Processing, v. 44, pp. 87-116, 1988. 
[18] M. Ohlsson, C. Peterson. Track finding with deformable templates, Computer Physics Communication, v. 71, pp. 77-98, 1992.

[19] J. Illingworth. The adaptive Hough transform, Trans. Pattern Analysis and Machine Intelligence, v. 9, n. 5, pp. 690-697, 1987.

[20] K. Sam Shamugan, A.M. Breipohl. Random Signals - detection, estimation and data analysis, Wiley, 2001.

[21] H.L.Van Trees. Detection, Estimation, and Modulation Theory, Part III, Wiley, 2001.

[22] A. Hyvarinen, J. Karhunen. Independent Component Analysis, Wiley, 2001.

[23] I. T. Jolliffe. Principal Component Analysis, Springer, 2002. 